Entropy 2006, 8[2], 63-66

Full paper

\title{
Entropy and Energy in Quantum Measurement
} Andreas E. Schlatter

Center for Physics Studies, Burghaldeweg 2, 5024 Küttigen, Switzerland

E-mail: andreas.schlatter@ubs.com

Received: 24 February 2006/Accepted: 10 May 2006/ Published: 12 May 2006

Abstract: On the basis of the classical axioms of non relativistic quantum mechanics, we develop a model for the interplay between energy and entropy in the process of quantum measurement and shed light on the scope of some of the axioms with regard to the measurement problem.

Keywords: Quantum Entropy, Energy, Measurement

MSC 2000 codes: 94A17, 81P15 


\section{Introduction}

In classical, non relativistic quantum mechanics a number of axioms or postulates were formulated at an early stage to clearly define the basis of the theory. As a reminder we state them in the following form:

1) The state of a physical system can be represented by a vector $|\psi\rangle$ in an abstract Hilbert space $\mathcal{H}$.

2) Every quantity $\mathcal{O}$ which can be observed is ascribed a hermitian operator $O$ acting on $\mathcal{H}$.

3) Only the eigenvalues of $O$ are possible results of a single measurement of the corresponding observable $\mathcal{O}$.

4) When an observable $\mathcal{O}$ is measured on a system $|\psi\rangle$ the probability $P\left(a_{n}\right)$ of obtaining the non-degenerate eigenvalue $a_{n}$ of the corresponding operator $O$ is

$$
P\left(a_{n}\right)=\frac{\left\langle u_{n} \mid \psi\right\rangle}{\langle\psi \mid \psi\rangle} ; \quad O\left|u_{n}\right\rangle=a_{n}\left|u_{n}\right\rangle, \quad n \in \mathbb{N}
$$

$5)$ If the measurement of the physical quantitiy $\mathcal{O}$ on the system in the state $|\psi\rangle$ gives the result $a_{n}$ the state of the system upon the measurement is the eigenvector $\left|u_{n}\right\rangle$.

6) The time evolution of the state vector $|\psi\rangle$ is governed by the Schroedinger equation to the given Hamiltonian $H$

$$
i \hbar \frac{\partial}{\partial t}|\psi\rangle=H|\psi\rangle
$$

Axiom 3) and the projection postulate 5) state that upon measurement there is a collapse of the wave function $|\psi\rangle$ to an eigenstate of the observable. The projection postulate is in conflict with axiom 6) which states a smooth evolution in the non-measurement case. In a nutshell, this conflict and its consequences forms the measurement problem which has been an urgent conceptual question in quantum mechanics ever since. In the present paper we combine ideas around the decoherence of states [7] and the insight that next to energy the "bookkeeping" of another quantitiy, namely entropy (information), plays an important role in the dynamics [8]. We will propose a simple model for the interplay between energy and entropy during measurement and derive some forceful consequences.

\section{$2 \quad$ The Model}

\subsection{Decoherence}

Let us start for our purposes with the simplest discussion of a single act of decoherence involving the measured system $\mathcal{S}$, the apparatus $\mathcal{A}$ and the environment $\mathcal{E}$ supposed to be one bit systems each. Let $\psi=\alpha|1\rangle+\beta|2\rangle$ be the measured state which interacts at first with the apparatus in the form of

$$
\begin{aligned}
& |1\rangle\left|A_{0}\right\rangle \rightarrow|1\rangle\left|A_{1}\right\rangle \\
& |2\rangle\left|A_{0}\right\rangle \rightarrow|2\rangle\left|A_{0}\right\rangle
\end{aligned}
$$


assuming that $\langle 1 \mid 2\rangle=\left\langle A_{0} \mid A_{1}\right\rangle=0$. Generally

$$
(\alpha|1\rangle+\beta|2\rangle)\left|A_{0}\right\rangle \rightarrow \alpha|1\rangle\left|A_{1}\right\rangle+\beta|2\rangle\left|A_{0}\right\rangle=\Phi .
$$

The environment in turn performs a premeasurement on the apparatus and as a result we get

$$
\left(\alpha|1\rangle\left|A_{1}\right\rangle+\beta|2\rangle\left|A_{0}\right\rangle\right)\left|\varepsilon_{0}\right\rangle \rightarrow \alpha|1\rangle\left|A_{1}\right\rangle\left|\varepsilon_{1}\right\rangle+\beta|2\rangle\left|A_{0}\right\rangle\left|\varepsilon_{0}\right\rangle=\Psi .
$$

It can be shown that any basis ambiguity of the correlated $\mathcal{A S}$ system can be overcome, if the environment satisfies $\left\langle\varepsilon_{0} \mid \varepsilon_{1}\right\rangle=0[7]$.

The description of the $\mathcal{A S}$ pair can now be readily obtained in terms of the reduced density matrix

$$
\left.\rho_{\mathcal{A S}}=\operatorname{tr}_{\mathcal{E}} \Psi\right\rangle\left\langle\Psi=|\alpha|^{2} \mid 1\right\rangle\left\langle 1|| A_{1}\right\rangle\left\langle\left. A_{1}|+| \beta\right|^{2} \mid 2\right\rangle\left\langle 2|| A_{0}\right\rangle\left\langle A_{0}\right| .
$$

This reduced density matrix only contains classical correlations and no more off-diagonal terms. Note that the $\mathcal{A S}$ system is an open quantum system and that the process of environmental decoherence is not governed by axiom 6) within $\mathcal{A S}$. Even by including the environment and the corresponding interaction Hamiltonians it is strictly speaking not possible to explain total decoherence by axiom 6). This can practically be explained by the fact that the number of interacting degrees of freedom is so big that only approximations of a Hamiltonian can be found. Decoherence is thus paractically irreversible.

\subsection{Energy, Entropy and Collapse}

The entropy of a quantum system can be defined in terms of the von Neumann entropy of its density matrix $\rho, S_{\rho}=-\operatorname{tr} \rho \ln \rho$. The entropy of pure states is zero. Hence in the above model there holds $S_{\rho_{\Phi}}=0$. At the same time $S_{\rho_{\mathcal{A S}}}=-\left(|\alpha|^{2} \ln |\alpha|^{2}+|\beta|^{2} \ln |\beta|^{2}\right)>0$. The interaction and energy exchange with the environment has thus increased the entropy of the $\mathcal{A S}$ system. This fact can be interpreted as a creation of classical possibilities with probability amplitudes $(\alpha, \beta)[9]$. It can classically best be compared to the expansion of some abstract phase space. From another angle one can say that information about the $\mathcal{A S}$ system has gone into the environment [9]. $\rho_{\mathcal{A S}}$, being a mixture of states, represents this collection of classical possibilities. According to postulate 5 ), one of these possibilities has to be realized and $\rho_{\mathcal{A S}}$ collapses into one of the eigenstates. This collapse of $\rho_{\mathcal{A S}}$ in turn reduces again the possibilities or compresses, to use the classical analogy, the abstract phase space.

We note that the measurement is according to postulate 5) a true erasure of information since after the fact no record is left of the original possibilities. If we assume that the environment is in termal equilibrium of temperature $T$, Landauer's principle $[4,5,10]$ tells us that the process is dissipative and a minimal average amount of energy $\bar{E}=k T S_{\rho_{\mathcal{A S}}}$ is being dissipated. By our model the measurement process is consequently irreversible.

\section{View on the Axioms}

Under the assumption of an open system, interacting with the environment, and of postulate 5) we see that the measurement process is an interplay between energy and entropy. In a first step 
the energy exchange through interaction with the environment leads to the decoherence of the $\mathcal{A S}$ system and a corresponding increase of entropy. This entropy increase reflects the expansion of some abstract phase space and the corresponding creation of classical alternatives. In a second step there is the collapse or realization of the decohered state which compresses phase space and reduces entropy again. In our model a logically irreversible operation is completed on the $\mathcal{A S}$ system and the measurement process turns out to be thermodynamically irreversible [10].

Under the assumption of a closed system with known Hamiltonian, however, the Schroedinger equation, axiom 6), is valid and due to the unitary nature of the evolution, the energy of the $\mathcal{A S}$ system is preserved, states are pure and entropy remains zero. The evolution governed by this process turns out to be reversible. From this angle it seems that axiom 6) is fully valid in the very special situation where a system is closed and the Hamiltonian can be known. According to our model, the measurement process seems to escape exact description by axiom 6). Postualte 5) plays an important role to understand reality and deserves to be listed on equal level with 6 ). Postualte 5) does not refer to time and the collapse seems instantaneous. Given the fact that our model predicts that a minimal average amount of heat is dissipated during the measurement process it is likely that measurement takes some minimal amount of time to happen. A natural next question would then be to ask "how long" it minimally takes to measure a quantum state.

\section{References}

1. Y. Aharonov, S.Messar, S.Popescu, "Measuring energy, estimating Hamiltonians, and the time-energy uncertainty relation", Phys. Rev. A 66, 052107, 2002

2. J. Bell, "On the Einstein Podolsky Rosen paradox", Physics 1, 3, 195, 1964

3. J. Bell, "On the problem of hidden variables in quantum mechanics", Rev. Mod. Phys. 38, 3, 447, 1966

4. R. Landauer, "Irreversibility and heat generation in the computing process", IBM J.Res.Dev. 5, 183-1991, 1961

5. B. Piechocinska, "Information erasue", Phys. Rev. A 61, 62314, 1-9, 2000

6. W. Zurek,"Maxwell's demon, Szilard's engine and quantum measurements", Frontiers of Nonequilibrium Statistical Physics, Plenum Press New York, 151-161, 1984a

7. J.P. Paz, W.H.Zurek, "Environment -Induced Decoherence and the Transition from Quantum to Classical" Course Lectures, 72nd Les Houches Summer School, July-August 1999

8. Todd L. Duncan, Jack S. Semura, "The Deep Physics Behind the Second Law:Information and Energy As independent Forms of Bookkeeping", Entropy 2004, 6, 21-29.

9. Jean-Bernard Brissaud, "The meanings of entropy", Entropy 2005, 7, 68-96

10. J. Ladyman, S. Presnell, A.J. Short, B. Groisman, "The Connection between Logical and Thermodynamic Irreversibility", preprint philsci-arXive ID 26892006

(C)2006 by MDPI (http://www.mdpi.org). Reproduction for noncommercial purposes permitted. 